\title{
A Role for Notch Signalling in Breast Cancer and Endocrine Resistance
}

\author{
Ahmet Acar, ${ }^{1}$ Bruno M. Simões, ${ }^{2}$ Robert B. Clarke, ${ }^{2,3}$ and Keith Brennan ${ }^{1}$ \\ ${ }^{1}$ Faculty of Life Sciences, University of Manchester, Oxford Road, Manchester M13 9PT, UK \\ ${ }^{2}$ Breast Biology Group, Institute of Cancer Sciences, University of Manchester, Wilmslow Road, Manchester M20 4BX, UK \\ ${ }^{3}$ Breast Cancer Now Research Unit, Institute of Cancer Sciences, University of Manchester, Wilmslow Road, Manchester M20 4BX, UK \\ Correspondence should be addressed to Keith Brennan; keith.brennan@manchester.ac.uk
}

Received 30 June 2015; Accepted 20 December 2015

Academic Editor: Armand Keating

Copyright (C) 2016 Ahmet Acar et al. This is an open access article distributed under the Creative Commons Attribution License, which permits unrestricted use, distribution, and reproduction in any medium, provided the original work is properly cited.

Over the past decade, there has been growing interest in the Notch signalling pathway within the breast cancer field. This interest stemmed initially from the observation that Notch signalling is aberrantly activated in breast cancer and its effects on various cellular processes including proliferation, apoptosis, and cancer stem cell activity. However more recently, elevated Notch signalling has been correlated with therapy resistance in oestrogen receptor-positive breast cancer. As a result, inhibiting Notch signalling with therapeutic agents is being explored as a promising treatment option for breast cancer patients.

\section{The Notch Signalling Pathway}

The Notch signalling pathway is evolutionarily conserved and is found in organisms as diverse as hydra and humans. In mammals, there are four Notch receptors (Notch1 to Notch4) and five DSL ligands (Jagged1, Jagged2, Delta-likel (Dll1), Dll3, and Dll4) [1]. As the DSL ligands are transmembrane proteins, Notch signalling is initiated by the interaction of DSL ligands and Notch receptors on adjacent cells. Their interaction leads to a proteolytic cleavage of the Notch protein at the S2 cleavage site mediated by ADAM10 and ADAM17. Following this cleavage, the remaining part of the Notch protein undergoes another proteolytic cleavage mediated by the $\gamma$-secretase enzyme complex. The latter cleavage releases the Notch intracellular domain (NICD), which translocates to the nucleus. Within the nucleus, NICD forms a complex with the DNA binding protein $\mathrm{RBPj}$ and a member of the Mastermind-like (MAML) family transcriptional coactivators (Figure 1). This complex of proteins then activates the transcription of Notch target genes, including members of Hes and Hey family of transcription. Other target genes include several genes that are directly associated with tumourigenesis such as cyclinD1 and Slug $[2,3]$. Notch signalling activity is also regulated through posttranslational modification. For example, Notch receptors are glycosylated through the sequential action of peptideO-fucosyltransferase (POFUT1) [4] and the Fringe GlcNAc transferases [5]; this modification alters the affinity of Notch for Delta and Serrate/Jagged ligands. Notch proteins are also subject to phosphorylation by glycogen synthase kinase $3 \beta$ $(\mathrm{GSK} 3 \beta)[6]$ and ubiquitination by the E3 ubiquitin ligase FBXW7 [7]. These latter modifications alter the stability of NICD and thereby control the duration of signalling.

\section{Notch Signalling Is Aberrantly Activated in Breast Cancer}

Breast cancer is one of the leading causes of death seen in the world despite the fact that there are ongoing efforts to improve detection and treatment. Breast cancer is a heterogeneous disease and currently is split into three subtypes clinically based on immunohistochemical analysis: $\mathrm{ER} \alpha+\mathrm{ve}$ (oestrogen receptor $\alpha$-positive), HER2 +ve (human epidermal growth factor receptor 2-positive), and triple negative (cancers that lack expression of ER $\alpha$, progesterone receptor (PR), and HER2). Currently, targeted therapies are available for ER $\alpha$ and Her2 +ve tumours. In addition to traditional subtype analysis, breast cancer can also be subtyped based on gene expression profiles. Perou and colleagues reported 


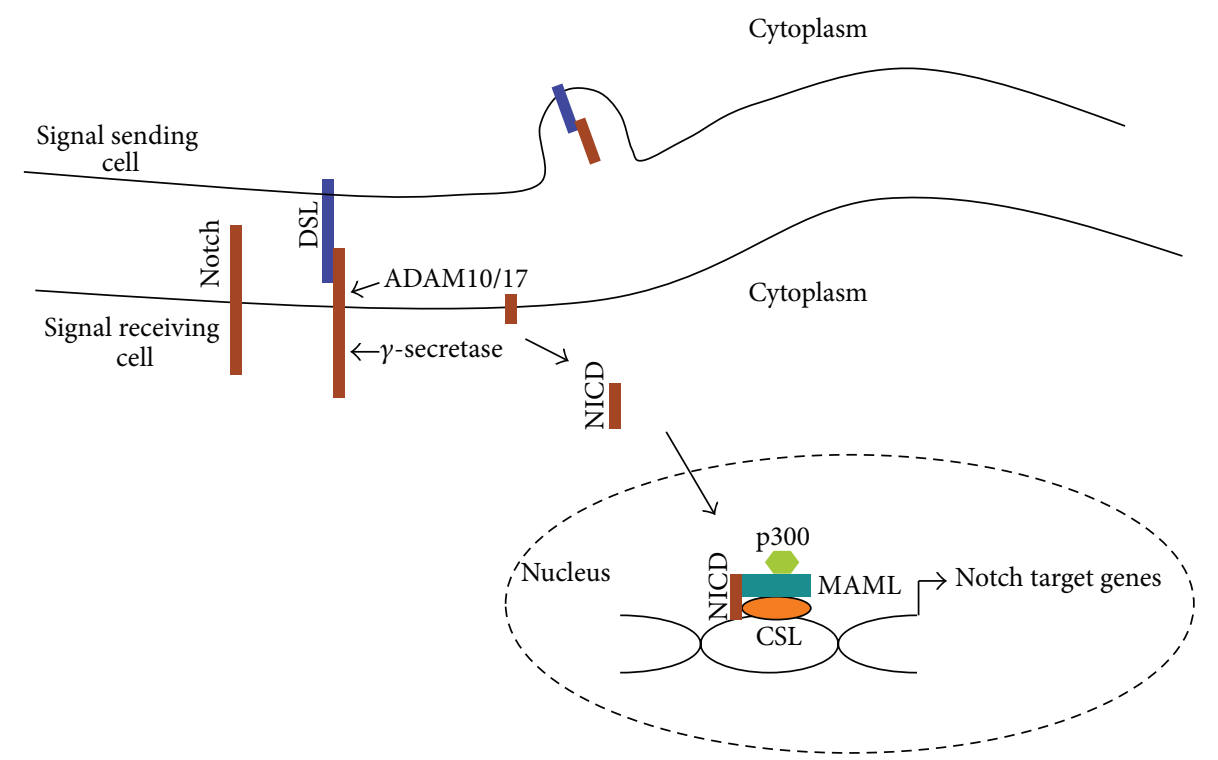

Figure 1: Basics of Notch signalling pathway. Notch signalling activation occurs via an interaction between DSL ligand and Notch receptor on adjacent cells. This interaction leads to force being applied to the extracellular domain of Notch, as DSL ligand undergoes endocytosis into the signalling cell, leading to a conformational change within the negative regulatory region (NRR). This conformational change exposes a cleavage site (S2) for the ADAM10 and ADAM17 proteases. The extracellularly truncated Notch protein then undergoes cleavage at site 3 (S3), mediated by $\gamma$-secretase, which releases the Notch intracellular domain (NICD). Finally, NICD translocates into the nucleus and interacts with the DNA binding protein RBPj and the transcriptional coactivators MAML and p300 to initiate transcription of downstream targets including the Hes and Hey family of genes.

the presence of luminal-like, normal-like, basal-like, and Her2-enriched subtypes [8]. They also split the luminal-like subtype into luminal A and luminal B subtypes. Although not perfectly aligned, the luminal-like, Her2-enriched, and basal-like subtypes correlate with ER $\alpha+v e$, HER2 +ve, and triple negative breast cancer subtypes identified by immunohistochemistry. More recently a sixth molecular subtype has been recognised, the claudin-low subtype [9]. Alternative molecular subtypes that link to copy number aberration data have also been proposed [10].

Over the past decade, aberrant activation of Notch signalling in breast cancer has been reported by many different groups. In invasive breast cancer, the elevated expression of Notch signalling pathway components has been reported, including Jagged1-2, Dll1, Dll3, and Dll4, Notch receptors, and Hes and Hey target genes [11]. For example, elevated expression of Jaggedl and Notchl has been linked to poor prognosis in breast cancer patients [12-14]. Likewise, Numb, a negative regulator of Notch signalling, has been found to be lost in $50 \%$ of breast cancer through ubiquitination and proteasomal degradation [15]. The accumulation of NICD has also been reported [16].

There are various examples of elevated Notch signalling being associated with a particular subtype of breast cancer and response to targeted therapy. For example, Notch signalling has been shown to be activated in ER $\alpha$ +ve breast cancer in response to treatment $[17,18]$. Elevated Notch1 expression is also found in HER2 +ve [19] and triple negative/basal breast tumours [13]. In contrast, elevated Notch2 is associated with highly differentiated and poorly proliferative breast cancers [20]. In fact, Notch signalling activation has even been observed in preinvasive breast lesions, including usual ductal hyperplasia (UDH) and ductal carcinoma in situ (DCIS) $[11,19,21]$. Lastly, a very recent study has identified a series of activating mutations within the PEST domain of Notch1, Notch2, and Notch3 [22]. These mutations were enriched in triple negative breast cancers.

The tumour promoting activity of Notch is evident from transgenic mouse model studies. For example, Notch4 intracellular domain when expressed under the control of the whey acidic promoter (WAP) or Notch1, Notch3, or Notch4 intracellular domains under the control of the mouse mammary tumour virus (MMTV) promoter all cause tumour formation in mice [23-26]. Also, conditional deletion of Lunatic Fringe in the mammary gland leads to elevated Jagged1-induced Notch signalling and formation of basal-like mammary tumours [27].

\section{Notch Signalling Regulates Many Cellular Properties of Breast Cancer}

Notch signalling is known to regulate many cellular processes including proliferation [17, 28], apoptosis [16, 29], angiogenesis [30,31], hypoxia [32], cancer stem cell activity [21, 33, 34], epithelial-to-mesenchymal transition (EMT) [3], and metastasis [35]. Notch signalling promotes proliferation in breast cancer cell lines by upregulating $\operatorname{cyclin} A, \operatorname{cyclin} B$, and cyclinD1 expression $[2,17,36]$. Notch protects breast epithelial cells from apoptosis by activating Akt [16, 29, 37]. It is thought that activation of Akt by Notch in this context occurs 
through downregulation of PTEN expression or secretion of an autocrine signalling protein $[29,36]$. Jagged1, expressed in tumour cells, has been shown to activate Notch signalling in neighbouring endothelial cells to promote angiogenesis [30]. Notch signalling can also regulate angiogenesis by limiting the number of tip cells formed and by promoting the arterial cell fate [31]. Notch signalling also regulates the self-renewal of breast cancer stem cells [21,34]. In this role, Notch4 appears to be particularly important, as knockdown of Notch4 has a much more significant effect on breast cancer stem cell numbers than Notchl knockdown [34]. Notch activity induces EMT by means of RBPj binding to promoter sequences upstream of the Slug gene [3]. Lastly, Notch activity has been shown to induce metastasis of breast cancer cells to the bone [35]. In this elegant study, the breast cancer cell line MDA-MB-231 was forced to express the Notch ligand Jagged1, and this was found to significantly increase bone metastasis [35]. Altogether, it is very clear that activation of Notch plays a key role in breast cancer. Therefore it represents a very attractive therapeutic target.

\section{Endocrine Therapy and Targeting Notch in Breast Cancer}

Current breast cancer treatments include general chemotherapeutic drugs such as epirubicin, doxorubicin, paclitaxel, and docetaxel, each aiming at blocking the proliferation of breast cancer cells. On the other hand, targeted therapies aim to disrupt the function of a protein or pathway known to drive tumour growth. Three out of four breast cancers express the oestrogen receptor (ER) alpha, and most of these depend on oestrogen for growth [38]. Consequently, targeting the function of ER is one of the most effective approaches to treat breast cancer [39]. Therapies against ER $\alpha+$ ve breast cancer include antioestrogens and aromatase inhibitors (AIs) [40]. Tamoxifen, which antagonises the action of ER by competing for the ligand-binding domain of the receptor, is the prevalent endocrine therapy of choice [41]. Other antioestrogens, such as pure antioestrogens or AIs, are considered to be a good choice when tamoxifen treatment fails [42]. AIs are also used in postmenopausal women without ovarian function and act by blocking the synthesis of oestrogen from androgens in the peripheral tissues [43]. Pure antioestrogens, like fulvestrant, block dimerization of the receptor and lead to ubiquitination and proteasome-mediated degradation of the receptor [44].

Unfortunately, ER-positive tumours frequently develop resistance to endocrine treatments and relapse. Although downregulation of ER expression can contribute to endocrine resistance, loss of the receptor during the acquisition of resistance is not commonly observed. Several recent studies have suggested that ER mutations, such as a tyrosine to asparagine substitution at residue 537 (Y537N), may occur during endocrine treatment $[45,46]$. These mutations activate $\mathrm{ER}$ and as a consequence lead to a loss of endocrine therapy responsiveness. The overexpression of different growth factor receptors and the activation of their downstream signalling pathways are also involved in the acquisition of endocrine resistance. Tumours resistant to tamoxifen are often associated with high expression and enhanced activity of tyrosine kinase receptors EGFR and HER2 [47]. These receptors activate kinases, such as ERK1/2, PI3K, and AKT, which promote phosphorylation and ligand independent activation of ER [38].

Over the past few years, there is accumulating evidence to suggest that cells with stem cell properties are the main driving force of tumourigenesis. Al-Hajj and colleagues were the first to show that a subpopulation of breast cancer cells, defined by a CD44+/ESA+/CD24lo cell surface phenotype, was capable of recapitulating the original tumour phenotype when transplanted into nonobese diabetic/severe combined immunodeficient (NOD/SCID) mice [48]. Since then, several groups have shown that breast tumours behave like an aberrant version of normal tissue and organise themselves into a cellular hierarchy with a cancer stem-like cell (CSC) at its apex. Indeed, expression of aldehyde dehydrogenase (ALDH), a stem/progenitor cell marker, has also been used to enrich for tumour-initiating CSCs [49]. The concept of CSCs has significant clinical implications, because these cells are believed to be responsible for tumour initiation and growth and are often resistant to chemo- and radiotherapy. In breast cancer, CD44+/ESA+/CD24lo cells are relatively insensitive to conventional chemotherapy and to radiation $[50,51]$.

Regarding endocrine therapy, there is accumulating evidence to suggest that an increase in breast CSCs occurs following endocrine therapy for ER $\alpha+v e$ tumours [52]. For example, Creighton and colleagues saw enrichment of cells with breast CSC features in tumour tissue derived from patients following therapy with an aromatase inhibitor (letrozole) [53]. Four other studies showed enrichment for CSC populations in ER $\alpha+$ ve breast cancer cell lines after tamoxifen treatment or oestrogen deprivation [18, 54-56]. The potential involvement of CSCs in breast cancer makes it imperative to further characterize these cells in order to find cellular signalling pathways that can be targeted to eradicate breast CSCs and, therefore, provide long-term disease-free survival.

One strong candidate pathway in this regard is the Notch pathway [21]. Pharmacologic and genetic inhibition of the Notch signalling can reduce breast CSC activity in vitro and tumour formation in vivo. Notch4 plays a particular key role in controlling breast CSCs [34]. In addition, it has also been reported that overexpression of specific Notch receptors is associated with treatment resistance in human breast cancer [57]. Likewise, it was shown that the use of antioestrogens can activate Notch signalling in breast cancer cells [17]. More recently Notch pathway was found to be hyperactivated in endocrine resistant breast cancer cells, and its inhibition blocked growth of these cells $[58,59]$. Moreover, we recently demonstrated that increased JAG1-NOTCH4 signalling in human breast tumours is an important driver of cancer stem cells [56].

Besides Notch signalling, other CSC pathways are aberrantly activated in endocrine resistant cells. For example, Wnt and Hedgehog signalling pathways are active in CSCs and this has already been shown to promote tamoxifen resistance $[55,60]$. Together these results suggest that inhibiting CSC signalling pathways will help to overcome endocrine therapy resistance and recurrence in $\mathrm{ER} \alpha+\mathrm{ve}$ breast cancer. 


\section{Conclusion}

It is widely recognized that increased Notch signalling is one of the main drivers of cellular malignancies in breast cancer. Moreover, Notch pathway activation is commonly seen in response to targeted therapies. Therefore, combining current treatment options with a blockade of Notch signalling might be a feasible approach to consider. With this being said, individual Notch receptors are likely to regulate mammary epithelial and breast cancer cells in unique ways; hence it is vital to delineate the functional role for individual Notch receptors in mediating resistance to therapy in breast cancer.

\section{Conflict of Interests}

The authors declare no conflict of interests.

\section{Acknowledgment}

The authors would like to thank Breast Cancer Now for their support of their laboratories.

\section{References}

[1] P. Ranganathan, K. L. Weaver, and A. J. Capobianco, "Notch signalling in solid tumours: a little bit of everything but not all the time," Nature Reviews Cancer, vol. 11, no. 5, pp. 338-351, 2011.

[2] B. Cohen, M. Shimizu, J. Izrailit et al., "Cyclin D1 is a direct target of JAG1-mediated Notch signaling in breast cancer," Breast Cancer Research and Treatment, vol. 123, no. 1, pp. 113124, 2010.

[3] K. G. Leong, K. Niessen, I. Kulic et al., "Jagged1-mediated Notch activation induces epithelial-to-mesenchymal transition through Slug-induced repression of E-cadherin," The Journal of Experimental Medicine, vol. 204, no. 12, pp. 2935-2948, 2007.

[4] S. Shi and P. Stanley, "Protein O-fucosyltransferase 1 is an essential component of Notch signaling pathways," Proceedings of the National Academy of Sciences of the United States of America, vol. 100, no. 9, pp. 5234-5239, 2003.

[5] V. M. Panin, V. Papayannopoulos, R. Wilson, and K. D. Irvine, "Fringe modulates Notch-ligand interactions," Nature, vol. 387, no. 6636, pp. 908-912, 1997.

[6] L. Espinosa, J. Inglés-Esteve, C. Aguilera, and A. Bigas, "Phosphorylation by glycogen synthase kinase- $3 \beta$ down-regulates Notch activity, a link for Notch and Wnt pathways," The Journal of Biological Chemistry, vol. 278, no. 34, pp. 32227-32235, 2003.

[7] S. Le Bras, N. Loyer, and R. Le Borgne, "The multiple facets of ubiquitination in the regulation of notch signaling pathway," Traffic, vol. 12, no. 2, pp. 149-161, 2011.

[8] C. M. Perou, T. Sørile, M. B. Eisen et al., "Molecular portraits of human breast tumours," Nature, vol. 406, no. 6797, pp. 747-752, 2000.

[9] J. I. Herschkowitz, K. Simin, V. J. Weigman et al., "Identification of conserved gene expression features between murine mammary carcinoma models and human breast tumors," Genome Biology, vol. 8, no. 5, article R76, 2007.

[10] S.-J. Dawson, O. M. Rueda, S. Aparicio, and C. Caldas, "A new genome-driven integrated classification of breast cancer and its implications," The EMBO Journal, vol. 32, no. 5, pp. 617-628, 2013.
[11] S. Mittal, D. Subramanyam, D. Dey, R. V. Kumar, and A. Rangarajan, "Cooperation of Notch and Ras/MAPK signaling pathways in human breast carcinogenesis," Molecular Cancer, vol. 8, article 128, 2009.

[12] M. Reedijk, S. Odorcic, L. Chang et al., "High-level coexpression of JAG1 and NOTCH1 is observed in human breast cancer and is associated with poor overall survival," Cancer Research, vol. 65, no. 18, pp. 8530-8537, 2005.

[13] M. Reedijk, D. Pinnaduwage, B. C. Dickson et al., "JAG1 expression is associated with a basal phenotype and recurrence in lymph node-negative breast cancer," Breast Cancer Research and Treatment, vol. 111, no. 3, pp. 439-448, 2008.

[14] B. C. Dickson, A. M. Mulligan, H. Zhang et al., "High-level JAG1 mRNA and protein predict poor outcome in breast cancer," Modern Pathology, vol. 20, no. 6, pp. 685-693, 2007.

[15] S. Pece, M. Serresi, E. Santolini et al., "Loss of negative regulation by Numb over Notch is relevant to human breast carcinogenesis," Journal of Cell Biology, vol. 167, no. 2, pp. 215221, 2004.

[16] S. Stylianou, R. B. Clarke, and K. Brennan, "Aberrant activation of notch signaling in human breast cancer," Cancer Research, vol. 66, no. 3, pp. 1517-1525, 2006.

[17] P. Rizzo, H. Miao, G. D'Souza et al., "Cross-talk between notch and the estrogen receptor in breast cancer suggests novel therapeutic approaches," Cancer Research, vol. 68, no. 13, pp. 5226-5235, 2008.

[18] J. M. Haughian, M. P. Pinto, J. C. Harrell et al., "Maintenance of hormone responsiveness in luminal breast cancers by suppression of Notch," Proceedings of the National Academy of Sciences of the United States of America, vol. 109, no. 8, pp. 2742-2747, 2012.

[19] S. J. Zardawi, I. Zardawi, C. M. McNeil et al., "High Notch1 protein expression is an early event in breast cancer development and is associated with the HER-2 molecular subtype," Histopathology, vol. 56, no. 3, pp. 286-296, 2010.

[20] C. Parr, G. Watkins, and W. G. Jiang, “The possible correlation of Notch-1 and Notch-2 with clinical outcome and tumour clinicopathological parameters in human breast cancer," International Journal of Molecular Medicine, vol. 14, no. 5, pp. 779-786, 2004.

[21] G. Farnie, R. B. Clarke, K. Spence et al., "Novel cell culture technique for primary ductal carcinoma in situ: role of Notch and epidermal growth factor receptor signaling pathways," Journal of the National Cancer Institute, vol. 99, no. 8, pp. 616$627,2007$.

[22] K. Wang, Q. Zhang, D. Li et al., "PEST domain mutations in Notch receptors comprise an oncogenic driver segment in triple-negative breast cancer sensitive to a gamma-secretase inhibitor," Clinical Cancer Research, vol. 21, no. 6, pp. 1487-1496, 2015.

[23] D. Gallahan, C. Jhappan, G. Robinson et al., "Expression of a truncated Int3 gene in developing secretory mammary epithelium specifically retards lobular differentiation resulting in tumorigenesis," Cancer Research, vol. 56, no. 8, pp. 1775-1785, 1996.

[24] C. Hu, A. Diévart, M. Lupien, E. Calvo, G. Tremblay, and P. Jolicoeur, "Overexpression of activated murine Notchl and Notch 3 in transgenic mice blocks mammary gland development and induces mammary tumors," The American Journal of Pathology, vol. 168, no. 3, pp. 973-990, 2006.

[25] J. Robbins, B. J. Blondel, D. Gallahan, and R. Callahan, "Mouse mammary tumor gene int-3: a member of the notch gene family 
transforms mammary epithelial cells," Journal of Virology, vol. 66, no. 4, pp. 2594-2599, 1992.

[26] H. Kiaris, K. Politi, L. M. Grimm et al., "Modulation of notch signaling elicits signature tumors and inhibits Hrasl-induced oncogenesis in the mouse mammary epithelium," The American Journal of Pathology, vol. 165, no. 2, pp. 695-705, 2004.

[27] K. Xu, J. Usary, P. C. Kousis et al., "Lunatic fringe deficiency cooperates with the Met/Caveolin gene amplicon to induce basal-like breast cancer," Cancer Cell, vol. 21, no. 5, pp. 626-641, 2012.

[28] K. Li, Y. Li, W. Wu et al., "Modulation of notch signaling by antibodies specific for the extracellular negative regulatory region of NOTCH3," The Journal of Biological Chemistry, vol. 283, no. 12, pp. 8046-8054, 2008.

[29] O. Meurette, S. Stylianou, R. Rock, G. M. Collu, A. P. Gilmore, and K. Brennan, "Notch activation induces Akt signaling via an autocrine loop to prevent apoptosis in breast epithelial cells," Cancer Research, vol. 69, no. 12, pp. 5015-5022, 2009.

[30] Q. Zeng, S. Li, D. B. Chepeha et al., "Crosstalk between tumor and endothelial cells promotes tumor angiogenesis by MAPK activation of Notch signaling," Cancer Cell, vol. 8, no. 1, pp. 1323, 2005.

[31] J. Dufraine, Y. Funahashi, and J. Kitajewski, "Notch signaling regulates tumor angiogenesis by diverse mechanisms," Oncogene, vol. 27, no. 38, pp. 5132-5137, 2008.

[32] H. Harrison, L. Rogerson, H. J. Gregson, K. R. Brennan, R. B. Clarke, and G. Landberg, "Contrasting hypoxic effects on breast cancer stem cell hierarchy is dependent on er-a status," Cancer Research, vol. 73, no. 4, pp. 1420-1433, 2013.

[33] H. Harrison, B. M. Simões, L. Rogerson, S. J. Howell, G. Landberg, and R. B. Clarke, "Oestrogen increases the activity of oestrogen receptor negative breast cancer stem cells through paracrine EGFR and Notch signalling," Breast Cancer Research, vol. 15, no. 2, article R21, 2013.

[34] H. Harrison, G. Farnie, S. J. Howell et al., "Regulation of breast cancer stem cell activity by signaling through the Notch4 receptor," Cancer Research, vol. 70, no. 2, pp. 709-718, 2010.

[35] N. Sethi, X. Dai, C. G. Winter, and Y. Kang, "Tumor-derived JAGGED1 promotes osteolytic bone metastasis of breast cancer by engaging notch signaling in bone cells," Cancer Cell, vol. 19, no. 2, pp. 192-205, 2011.

[36] H. Ling, J.-R. Sylvestre, and P. Jolicoeur, "Notch1-induced mammary tumor development is cyclin D1-dependent and correlates with expansion of pre-malignant multipotent ductlimited progenitors," Oncogene, vol. 29, no. 32, pp. 4543-4554, 2010.

[37] T. P. Dang, "Notch, apoptosis and cancer," Advances in Experimental Medicine and Biology, vol. 727, pp. 199-209, 2012.

[38] S. Ali and R. C. Coombes, "Endocrine-responsive breast cancer and strategies for combating resistance," Nature Reviews Cancer, vol. 2, no. 2, pp. 101-112, 2002.

[39] C. K. Osborne and R. Schiff, "Mechanisms of endocrine resistance in breast cancer," Annual Review of Medicine, vol. 62, pp. 233-247, 2011.

[40] J. Dittmer and B. Leyh, "The impact of tumor stroma on drug response in breast cancer," Seminars in Cancer Biology, vol. 31, pp. 3-15, 2014.

[41] V. C. Jordan, "Selective estrogen receptor modulation: concept and consequences in cancer," Cancer Cell, vol. 5, no. 3, pp. 207213,2004
[42] A. Howell, J. F. R. Robertson, P. Abram et al., "Comparison of fulvestrant versus tamoxifen for the treatment of advanced breast cancer in postmenopausal women previously untreated with endocrine therapy: a multinational, double-blind, randomized trial," Journal of Clinical Oncology, vol. 22, no. 9, pp. 1605-1613, 2004.

[43] W. R. Miller, "Aromatase inhibitors: mechanism of action and role in the treatment of breast cancer," Seminars in Oncology, vol. 30, no. 4, pp. 3-11, 2003.

[44] R. W. Carlson, "The history and mechanism of action of fulvestrant," Clinical Breast Cancer, vol. 6, supplement 1, pp. S5S8, 2005.

[45] D. R. Robinson, Y.-M. Wu, P. Vats et al., "Activating ESR1 mutations in hormone-resistant metastatic breast cancer," Nature Genetics, vol. 45, no. 12, pp. 1446-1451, 2013.

[46] W. Toy, Y. Shen, H. Won et al., "ESR1 ligand-binding domain mutations in hormone-resistant breast cancer," Nature Genetics, vol. 45, no. 12, pp. 1439-1445, 2013.

[47] J. M. Gee, J. F. Robertson, E. Gutteridge et al., "Epidermal growth factor receptor/HER2/insulin-like growth factor receptor signalling and oestrogen receptor activity in clinical breast cancer," Endocrine-Related Cancer, vol. 12, supplement 1, pp. S99-S111, 2005.

[48] M. Al-Hajj, M. S. Wicha, A. Benito-Hernandez, S. J. Morrison, and M. F. Clarke, "Prospective identification of tumorigenic breast cancer cells," Proceedings of the National Academy of Sciences of the United States of America, vol. 100, no. 7, pp. 39833988, 2003.

[49] C. Ginestier, M. H. Hur, E. Charafe-Jauffret et al., "ALDH1 is a marker of normal and malignant human mammary stem cells and a predictor of poor clinical outcome," Cell Stem Cell, vol. 1, no. 5, pp. 555-567, 2007.

[50] X. Li, M. T. Lewis, J. Huang et al., "Intrinsic resistance of tumorigenic breast cancer cells to chemotherapy," Journal of the National Cancer Institute, vol. 100, no. 9, pp. 672-679, 2008.

[51] M. Diehn, R. W. Cho, N. A. Lobo et al., "Association of reactive oxygen species levels and radioresistance in cancer stem cells," Nature, vol. 458, no. 7239, pp. 780-783, 2009.

[52] B. M. Simões, D. G. Alferez, S. J. Howell, and R. B. Clarke, "The role of steroid hormones in breast cancer stem cells," EndocrineRelated Cancer, vol. 22, no. 6, pp. T177-T186, 2015.

[53] C. J. Creighton, X. Li, M. Landis et al., "Residual breast cancers after conventional therapy display mesenchymal as well as tumor-initiating features," Proceedings of the National Academy of Sciences of the United States of America, vol. 106, no. 33, pp. 13820-13825, 2009.

[54] B. M. Simões, M. Piva, O. Iriondo et al., "Effects of estrogen on the proportion of stem cells in the breast," Breast Cancer Research and Treatment, vol. 129, no. 1, pp. 23-35, 2011.

[55] M. Piva, G. Domenici, O. Iriondo et al., "Sox2 promotes tamoxifen resistance in breast cancer cells," EMBO Molecular Medicine, vol. 6, no. 1, pp. 66-79, 2014.

[56] B. Simões, C. O’Brien, R. Eyre et al., "Anti-estrogen resistance in human breast tumors is driven by JAG1-NOTCH4-dependent cancer stem cell activity," Cell Reports, vol. 12, no. 12, pp. 19681977, 2015.

[57] A. Magnifico, L. Albano, S. Campaner et al., "Tumor-initiating cells of HER2-positive carcinoma cell lines express the highest oncoprotein levels and are sensitive to trastuzumab," Clinical Cancer Research, vol. 15, no. 6, pp. 2010-2021, 2009. 
[58] L. Magnani, A. Stoeck, X. Zhang et al., "Genome-wide reprogramming of the chromatin landscape underlies endocrine therapy resistance in breast cancer," Proceedings of the National Academy of Sciences of the United States of America, vol. 110, no. 16, pp. E1490-E1499, 2013.

[59] J. Yun, A. Pannuti, I. Espinoza et al., "Crosstalk between PKC $\alpha$ and Notch-4 in endocrine-resistant breast cancer cells," Oncogenesis, vol. 2, article e60, 2013.

[60] B. Ramaswamy, Y. Lu, K.-Y. Teng et al., "Hedgehog signaling is a novel therapeutic target in tamoxifen-resistant breast cancer aberrantly activated by PI3K/AKT pathway," Cancer Research, vol. 72, no. 19, pp. 5048-5059, 2012. 

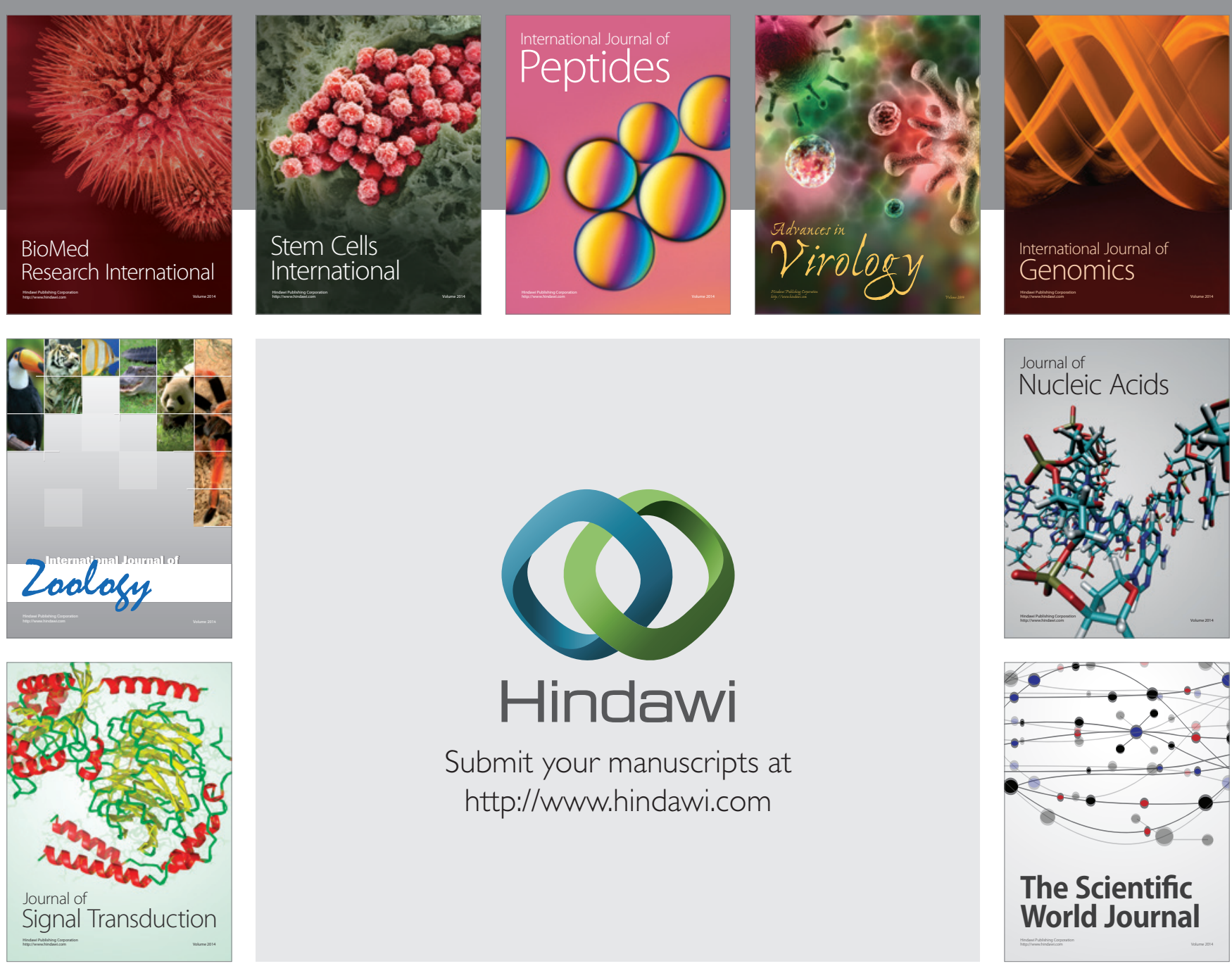

Submit your manuscripts at

http://www.hindawi.com
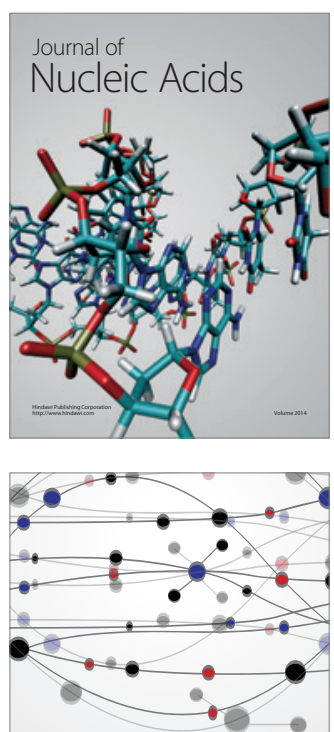

The Scientific World Journal
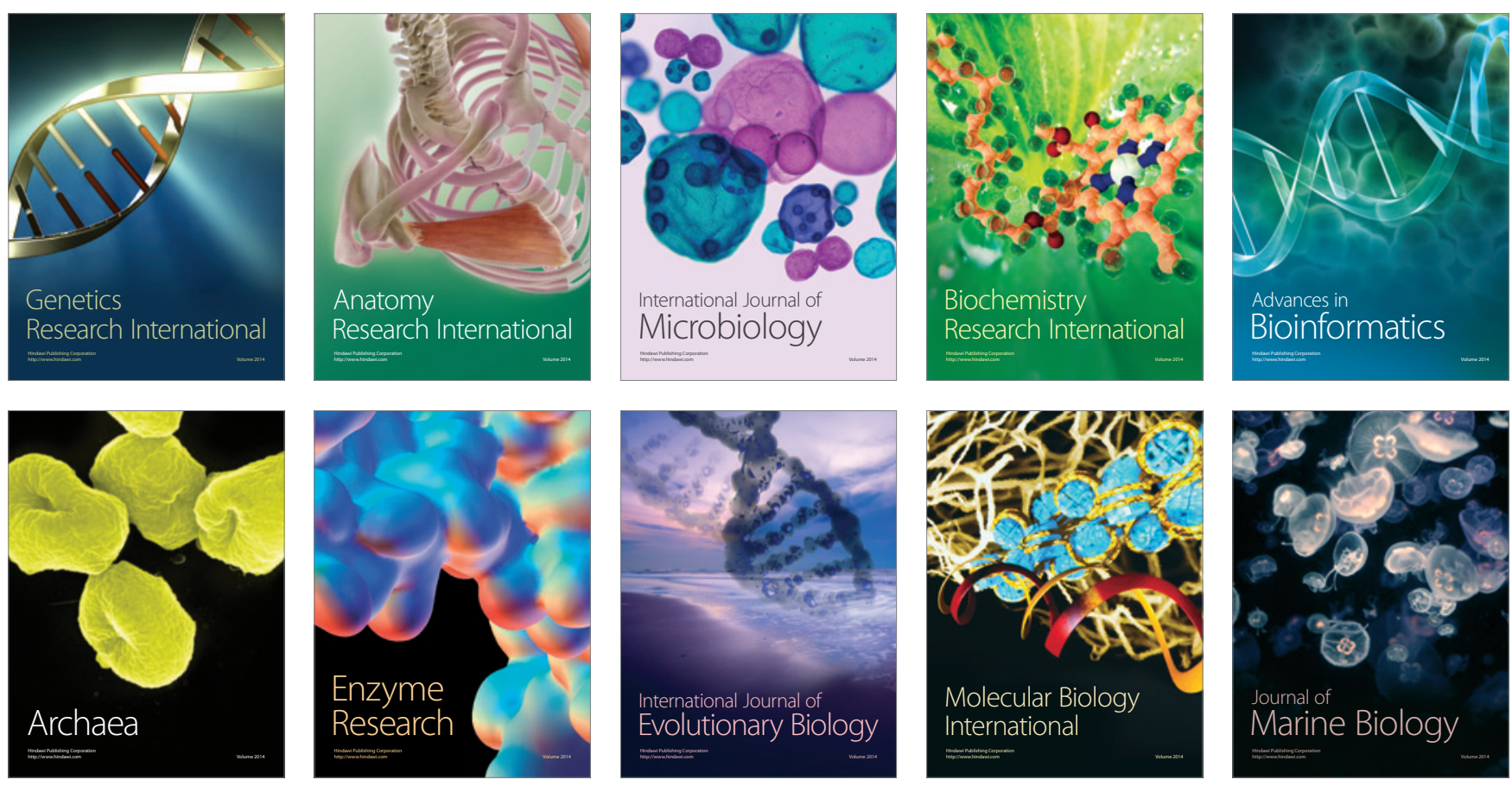